\title{
A retrospective study of biochemical parameters and outcomes of obstetric cholestasis: with and without pruritus
}

\author{
Nidhi Garg, Veena Ganju Malla \\ Corresponding author: Dr Veena Ganju Malla, Professor, Department of Obstetrics \& Gynecology \\ ABVIMS \& Dr. RML Hospital, New Delhi, India; Email - veenaganju@yahoo.com
}

Distributed under Attribution-Non Commercial - Share Alike 4.0 International (CC BY-NC-SA 4.0)

\begin{abstract}
Objectives: The objective was to study the differences in the biochemical parameters and outcomes of obstetric cholestasis with and without pruritus. Methods: This retrospective hospital based study was done from July 2014 to June 2018 where 160 confirmed cases of cholestasis were included in the study. The subjects were assessed for demographic parameters, obstetric history, and liver function tests, including bile acids. Results: Pruritus was seen in $128(80 \%)$ patients. There were significantly higher levels of serum bilirubin in the patients without pruritus; other parameters were comparable. Maternal outcome parameters were comparable. All the infants born to mothers in without pruritus group and $84.38 \%$ of those born to mothers with pruritus recovered uneventfully. No stillbirths and intrauterine deaths were reported. Conclusions: Among all Obstetric cholestasis patients, pruritus was not present in $20 \%$ of patients. Patients without pruritus had significantly higher serum bilirubin, while other parameters of liver function tests were statistically comparable to cholestasis women with pruritus. The cautious management of women without pruritus resulted in uneventful deliveries and comparable outcomes with those with pruritus.
\end{abstract}

Keywords: Intrahepatic cholestasis of pregnancy (IHCP), maternal outcome, obstetric cholestasis,

pruritus.

Cholestasis is the obstruction of bile outflow, causing raised serum bile acids and deranged liver function tests (LFTs). It is common in pregnancy, with a usual onset in the second or third trimester of pregnancy and spontaneous resolution within 2-3 weeks after delivery: termed as obstetric cholestasis or intrahepatic cholestasis of pregnancy (IHCP). ${ }^{1}$ Pruritus is a typical symptom of obstetric cholestasis and a known complication of generalized cholestasis. Women with cholestasis of pregnancy can experience severe pruritus, especially in the third trimester. The mechanism of pruritus has been linked with the active inflammation of the biliary epithelial cells causing the release of certain pruritogens. Thus, pruritus can persist and remit throughout the course of the disease depending upon the degree of biliary inflammation and the serum levels of pruritogens or its cofactors. ${ }^{2}$

This observation is interesting because we found that most of the second to third-trimester pregnant women at our institute with deranged LFT and raised serum bile acids did not present with pruritus. Thus we did a retrospective study to assess the prevalence of pruritus in pregnant women with deranged LFT and serum bile acids. The objective was to study the differences in the biochemical parameters and outcomes among women with and without pruritus.

\section{Methods}

The retrospective observational study was conducted

Received: $1^{\text {st }}$ December 2019. Accepted: $26^{\text {th }}$ February 2020.

Garg N, Malla VG. A retrospective study of biochemical parameters and outcomes of obstetric cholestasis: with and without pruritus. The New Indian Journal of OBGYN. 2020; 7(1): 88-92. 
from July 2014 to July 2018 in the department of Obstetrics and Gynecology at a tertiary care hospital. Patients were enrolled from the antenatal OPD (Out patient department) and labor ward of our unit. Eligible subjects were enrolled in the study only after obtaining informed written consent. As it was a retrospective study without any active intervention, institutional ethical clearance was not required.

The 5-year records were searched for those pregnant women who had abnormal liver function test (LFT) (raised AST and ALT) during 32-34 weeks. The ones with deranged liver function were looked for deranged serum bile acids and only those women who had both deranged LFTs and SBA (Serum bile acid) were included in the study. Any antenatal women visiting the OPD with complaints of pruritus were worked up for intrahepatic cholestasis of pregnancy (IHCP) after ruling out dermatological differentials of pruritus. The gestational age (GA) at which the patient complained of pruritus was noted. The evaluation for IHCP included measurement of LFTs, serum bile acid, ruling out viral hepatitis by viral markers, ultrasonography of the upper abdomen to evaluate the liver echotexture, coagulation profile.

The patients were followed as per their routine visits in the antenatal OPD with serial LFTs, SBA and symptom improvement after treating them with individualized doses of ursodeoxycholic acid ranging between $300 \mathrm{mg}$ to $1200 \mathrm{mg}$ divided doses till postpartum period. The patients diagnosed with IHCP were given the option of elective induction/ expectant management around 37 to 38 weeks gestation. Patients were induced after taking due consent and rest were under close fetomaternal monitoring till either spontaneous onset of labor or induced at lateterm.

Statistical analysis: Categorical variables were presented in number and percentage (\%) and continuous variables were presented as mean \pm SD. Quantitative variables were compared using independent t-test between the two groups. Qualitative variables were correlated using the Chi-Square test/Fisher's Table 3: Lab Parameters of IHCP Patients

\begin{tabular}{lll}
\hline Parameters & Mean & Standard Deviation \\
\hline Total Bilirubin (mg/dL) & 0.74 & 0.72 \\
SGOT (U/L) & 108 & 97.6 \\
SGPT (U/L) & 119.46 & 100.66 \\
ALP (U/L) & 235.9 & 125.43 \\
SBA (U/L) & 17.9 & 15.4 \\
\hline
\end{tabular}

SGOT $=$ Serum glutamic-oxaloacetic transaminase, $\mathrm{SGPT}=$ Serum glutamic pyruvic transaminase, $\mathrm{ALP}=$ Alkaline phosphatise, $\mathrm{SBA}=$ Serum bile acid exact test. A $p$ - value of $<0.05$ was considered statistically significant. The data was entered in MS EXCEL spreadsheet and analysis was done using Statistical Package for Social Sciences (SPSS) version 21.0.

\section{Results}

A total of 200 case records were scrutinized with altered LFTs. Table 1 shows the demographic characteristics of these subjects. In the present study, 63.5\% $(\mathrm{n}=127)$ were primipara with the majority being in the age group of 20 to 29 years. Forty case records were found to have deranged LFTs without raised serum bile acid levels and thus were not analyzed further $(27.7 \pm 4.04)$. A total of 160 patients who

Table 1: Demographic characteristics

\begin{tabular}{lll}
\hline Parity & Number of patients & Percentage \\
\hline Primipara & 127 & $63.5 \%$ \\
Multipara & 73 & $36.5 \%$ \\
\hline Age in years & & \\
\hline$<20$ & 1 & $0.5 \%$ \\
$20-29$ & 136 & $68 \%$ \\
$30-39$ & 62 & $31 \%$ \\
$>40$ & 1 & $0.5 \%$ \\
\hline
\end{tabular}

fitted in the criteria of IHCP were analyzed further for detailed clinical spectrum, course of pregnancy and fetomaternal outcome. Pruritus was observed in 128 (80\%) patients and was characteristically nocturnal and generally

Table 2: Period of gestation (POG) at the onset of pruritus in IHCP cases

\begin{tabular}{lll}
\hline $\begin{array}{l}\text { Gestational } \\
\text { Age(weeks) }\end{array}$ & Total & Percentage \\
\hline$<20$ weeks & 2 & 1.56 \\
$21-25$ weeks & 1 & 0.7 \\
26- 30 weeks & 19 & 14.84 \\
$31-35$ weeks & 61 & 47.65 \\
$>36$ weeks & 45 & 35.15 \\
\hline Total & $\mathbf{1 2 8}$ & $\mathbf{1 0 0 \%}$ \\
\hline
\end{tabular}

specific to palms and soles while 32 patients had an absence of pruritus till the termination of pregnancy. The onset of pruritus was observed to be in the second half of pregnancy, with only $2.25 \% \quad(n=3)$ having onset before 26 weeks 
profile of all patients was found normal with the mean INR value of $1.08 \pm 0.45$. All patients were treated with
$80 \%$ had pruritus, and $20 \%$ had no pruritus. We found that the maternal and neonatal outcome among the patients with
Table 4: Association of Pruritus with LFTs in IHCP patients

\begin{tabular}{|c|c|c|c|}
\hline Parameter (Mean \pm SD) & Pruritus absent & Pruritus Present & $P$ value \\
\hline Total bilirubin & $0.96 \pm 1.52$ & $0.68 \pm 0.29$ & 0.0002 \\
\hline SGOT & $125.7 \pm 125.02$ & $103.67 \pm 89.52$ & 0.107 \\
\hline SGPT & $124.25 \pm 109.22$ & $118.26 \pm 98.83$ & 0.96 \\
\hline ALP & $272.46 \pm 139.73$ & $251.56 \pm 121.66$ & 0.185 \\
\hline SBA & $18.35 \pm 11.32$ & $17.86 \pm 16.37$ & 0.073 \\
\hline
\end{tabular}

ursodeoxycholic acid and $18 \% \quad(\mathrm{n}=30)$ needed ursodeoxycholic acid in more than $900 \mathrm{mg}$ per day in divided doses. Various LFTs parameters were compared between two groups of IHCP patients. We found significantly higher serum bilirubin in the patients without pruritus; whereas other parameters were statistically comparable (table 4).

The majority of the patients were nullipara followed by primipara. GA in most of the patients was $>37$ weeks; mode

Table 5: Maternal outcome parameters

\begin{tabular}{llll}
\cline { 1 - 2 } Parameters & Pruritus absent & Pruritus Present & \multirow{2}{*}{ P value } \\
\cline { 1 - 3 } GA at delivery in weeks & & \\
\cline { 1 - 2 }$<37$ weeks & $6(18.75 \%)$ & $14(10.94 \%)$ & \\
$>37$ weeks & $26(81.25 \%)$ & $114(89.06 \%)$ & \\
\cline { 1 - 2 } Mode of delivery & & \\
\cline { 1 - 2 } Normal vaginal delivery & $20(62.50 \%)$ & $80(62.50 \%)$ & \\
LSCS & $12(37.50 \%)$ & $48(37.50 \%)$ & \\
\hline
\end{tabular}

of delivery in the majority was normal vaginal delivery (NVD) (table 5). Maternal outcome parameters, such as term/preterm gestation, and mode of delivery were comparable among both groups of IHCP patients $(\mathrm{p}>0.05)$.

Table 6: Neonatal outcome parameters

\begin{tabular}{llll}
\hline Parameters & Pruritus absent & Pruritus present & P value \\
\hline Still births & $0(0.00 \%)$ & $0(0.00 \%)$ & - \\
Intra uterine death & $0(0.00 \%)$ & $0(0.00 \%)$ & - \\
Uneventful & $32(100.00 \%)$ & $128(100 \%)$ & 1 \\
\hline
\end{tabular}

There were no cases of stillbirths and intrauterine death (IUD) in this study (table 6); all the infants born to mothers without pruritus and $100 \%$ of those born to mothers with pruritus recovered uneventfully.

\section{Discussion}

In the present study, we assessed maternal and fetal outcome in intrahepatic cholestasis of pregnancy and tried to know if the absence of pruritus can be ignored in diagnosing the patients for IHCP. Though IHCP is typically associated with pruritus, ${ }^{3}$ we found some interesting observations. On retrospectively analyzing the records, we found 160 patients with deranged LFT and raised serum bile acids, out of which
Pruritus is a subjective symptom and it may suffer from a reporting error/bias on the part of the lower middle class population that is mainly dealt in the government hospitals of India. The waxing and vaning property of pruritus may make it unlinkable to the pregnancy; resulting in non-reporting. Thirdly, pregnancy in itself encompasses so many different symptoms like nausea, vomiting, excessive urination, etc. that pruritus may be ignored when one visits an obstetrician for routine checkup.

Thus, any such patients (without pruritus) also must be regularly screened and monitored as those with pruritus for diagnosing any liver dysfunctions. This can be done by initiating routine LFT monitoring of the pregnant patients from the beginning of the second trimester to detect any abnormal LFT even in the absence of pruritus. The mean age of patients in our study was $27.7 \pm 4.04$ years. Similar age distribution (25-30 years) in the study subjects of IHCP has been reported by other studies. ${ }^{1,4,5}$ ICP typically presents with pruritus in the third trimester, and in approximately $80 \%$ of women, it presents after 30 weeks of gestation. However, there are reports of onset in the first trimester, and of earlier onset in women with multifetal pregnancy. ${ }^{6}$

In the present study, in most of the patients (97.75\%), the onset of pruritus was observed to be in the second half of pregnancy, i.e. after 26 weeks. In a study by Kant A et al, maximum subjects in the study showed the onset of symptoms (pruritus) at 32-36 weeks of pregnancy. IHCP is characterized by pruritus that starts in the 2 nd or $3 \mathrm{rd}$ trimester of pregnancy and disappears following delivery. ${ }^{1}$ Brouwers et al., reported that the diagnosis of pruritus was done at 33-36 weeks, which suggests similar findings for the onset of pruritus as that of our study. Moreover, the disease that presents late in the course of pregnancy was observed to 
be mild in severity. ${ }^{7}$ Women with pruritus should have deranged LFTs, including serum bile acid levels. It is suggested that women with pruritus but the normal liver function or normal serum bile acids should have their blood tests repeated after 1-2 weeks because the onset of pruritus can precede abnormal biochemistry. ${ }^{4}$ Similarly, a women without pruritus should also have routine blood testing for liver functions as pruritus may be absent at presentation or it may come in phases and depends on an individual basis. It must be noted during the routine monitoring that an increase in liver transaminases can occur before or after the increase in serum bile acids, and there is a poor correlation between the level of transaminases and the bile acid concentration. The most sensitive and specific marker for the diagnosis and monitoring of IHCP are serum bile acids; they can be used for identifying women at increased risk of adverse perinatal outcomes. Alanine aminotransferase (ALT) is considered to be more specific to the liver than aspartate aminotransferase (AST). ALP is not useful in the diagnosis or monitoring of this condition. ${ }^{4}$ Thus Pruritus can be kept in the optional category for diagnosing and managing women with IHCP.

In this study, the frequency of nullipara was highest followed by primipara. In majority, gestational was more than 37 weeks; in most of the patients, mode of delivery was normal vaginal delivery. There was no significant difference between both groups of IHCP patients in terms of maternal outcome parameters, like parity, GA at delivery in weeks, and mode of delivery. Results of the studies by Geenes et al. andShobailliet al. are in accordance with that of our study. ${ }^{4,5}$ Geenes et al. reported that there was no significant difference present between the parity of IHCP subjects and control subjects. ${ }^{4}$ In another study by Shobailli et al., the difference in mean parity did not reach a statistically significant difference. ${ }^{5}$ In contrast, In a study by Kant $\mathrm{A}$ et al., the number of primipara women was significantly higher in the IHCP with the Pruritus group than the non-pruritus group. ${ }^{1}$ This was in contrast to the general belief that IHCP is more commonly found in the multiparous women of advanced maternal age. GA was more than 37 weeks in the majority of the patients. GA at delivery was significantly higher in the control group as compared to those with pruritus. There was no significant difference in the mode of delivery in both groups. In a similar study by Dang A et al. conducted in an Indian setting, it was noted that the frequency of primipara was significantly higher in the IHCP group in comparison with controls, which indicates probably different presentation of IHCP in north India. ${ }^{8}$ This difference could be because of socio-demographic factors comprising preferences of the pregnant women for different hospital set up. In the present study, stillbirths and intrauterine deaths (IUDs) were not observed. All the infants born in without pruritus group and $84.38 \%$ born in with the pruritus group recovered uneventfully. In concordance to the results of our study, in the study by Kant et al., there were no stillbirth and IUD reported. ${ }^{1}$ Shobaili et al. also noted no significant difference in the rate of IUD among the two groups. ${ }^{5}$ Geenes et al. observed that there was a significantly higher rate of stillbirth in the IHCP group. ${ }^{4}$ Shemer et al. noted that there was no significant difference in both groups in terms of the frequency of neonatal death. ${ }^{9}$

IHCP may be associated with an increased risk of adverse perinatal outcomes, including spontaneous preterm birth, fetal distress during labor, preterm meconium staining of the amniotic fluid, and stillbirth but due to the differences in the diagnostic criteria for IHCP and the changes in obstetric management practices over time, it is difficult to define the true incidence of each adverse outcome and the precise nature of the risks for individual pregnancies. ${ }^{10}$

We found all uneventful deliveries among the women with and without pruritus, signifying the importance of the proper diagnosis and management of both the groups. We highly recommend that pruritus may be kept in the optional category for diagnosis of IHCP for Indian women based on the following factors:

- No significant difference in the maternal and perinatal outcome among the women with deranged LFT and bile acids but with/without pruritus

- Inadequate reporting of pruritus among the Indian women with poor socioeconomic status.

- Consideration of pruritus as an insignificant symptom and thus not linking it to pregnancy

Limitations and strengths of the study: Our study suffers from the limitation of being retrospective in nature, and thus cause-effect relationship could not be established with pruritus. However, the strengths of the study were that the women without pruritus were also considered in the group of IHCP and were managed in a better way beforehand with regular monitoring of their LFTs.

\section{Conclusion}

Among all IHCP patients, Pruritus was not present in $20 \%$ of patients. The cautious management of women without pruritus resulted in uneventful deliveries and comparable obstetric outcomes with those with pruritus. It may be recommended to consider the women without 
The New Indian Journal of OBGYN. 2020 (July-December); 7(1)

pruritus into the category of IHCP if they have deranged bile acids and LFTs and manage them cautiously in an equivalent manner to IHCP women with pruritus. However, further long term follow up comparative studies are needed to redefine the inclusion criteria of IHCP in terms of Pruritus

Acknowledgement: We thank Dr Ketan Garg for assistance in medical writing and editing.

\section{Conflict of interest: None. Disclaimer: Nil.}

\section{References}

1. Kant A, Goswami S, Gupta U, Razdan A, Amle D. Maternal and perinatal outcome in cholestasis of pregnancy: a study in tertiary care hospital in North India. Int J Reprod Contracept Obstet Gynecol. 2018; 7: 5066-70.

2. Bergasa NV. Itch: mechanisms and treatment. In: Carstens E, Akiyama T (Eds). Pruritus of Cholestasis. Boca Raton (FL): CRC Press/Taylor \& Francis; 2014. Available from https://www.ncbi.nlm.nih.gov/books/NBK200923/ [Accessed October, 2019].

3. Geenes V, Williamson C, Chappell LC. Intrahepatic cholestasis of pregnancy. Obstet Gynecol. 2016; 18: 273-81.

4. Geenes V, Chappell L, Seed P, Steer P, Knight M, Williamson C. Association of severe intrahepatic cholestasis of pregnancy with adverse pregnancy outcomes: A prospective population-based case-control study. Hepatol. 2014; 59(4): 1482-91.

5. Al Shobaili H, Hamed H, Al Robaee A, Alzolibani A, Amin A, Ahmad S. Obstetrical and fetal outcomes of a new management strategy in patients with intra-hepatic cholestasis of pregnancy. Arch Gynecol Obstet. 2010; 283(6): 1219-25.

6. Kenyon AP, Piercy CN, Girling J, Williamson C, Tribe RM, Shennan AH. Obstetric cholestasis, outcome with active management: a series of 70 cases. BJOG. 2002; 109: 282-8.

7. Brouwers L, Koster M, Page-Christiaens G, Kemperman H, Boon J, Evers I, et al. Intrahepatic cholestasis of pregnancy: maternal and fetal outcomes associated with elevated bile acid levels. Am J Obstet Gynecol. 2015; 212(1): 100.e1-7.

8. Dang A, Agarwal N, Bathla S, Sharma N, Balani S. Prevalence of liver disease in pregnancy and its outcome with emphasis on obstetric cholestasis: An Indian scenario. J Obstet Gynecol India. 2010; 60(5): 413-8.

9. Shemer WE, Marschall H, Ludvigsson J, Stephansson $\mathrm{O}$. Intrahepatic cholestasis of pregnancy and associated adverse pregnancy and fetal outcomes: a 12-year population-based cohort study. BJOG. 2013; 120(6): 717-23.

10. Glantz A, Marschall HU, Mattsson LA. Intrahepatic cholestasis of pregnancy: Relationships between bile acid levels and fetal complication rates. Hepatology. 2004; 40: 467-74.

Nidhi Garg ${ }^{1}$, Veena Ganju Malla ${ }^{2}$

${ }^{1}$ Senior Resident; ${ }^{2}$ Professor, Department of Obstetrics \&Gynecology, ABVIMS \&Dr. RML Hospital, New Delhi, India. 